\title{
Impulsive Noise Reduction Method Based on Clipping and Adaptive Filters in AWGN Channel
}

\author{
Sumrin M. Kabir, Alina Mirza, and Shahzad A. Sheikh
}

\begin{abstract}
Impulsive noise is a man-made non-Gaussian noise that may destroy the information badly. Previously, methods have been investigated to mitigate this noise. In this paper, an adaptive filter based impulsive noise cancellation technique is introduced, comprised of Normalized Least Mean Square (NLMS) and Recursive Least Square (RLS) algorithms along with clipping method. The proposed scheme is tested on binary data modulated over different types of constellation schemes. The performance of this method is quite better than the previously existing techniques in terms of bit error rates (BER) for different modulation schemes namely QPSK, 16 QAM and 32 QAM. The convergence characteristics are demonstrated by the simulation results comparing their Bit Error Rates (BER) under same parameters.
\end{abstract}

Index Terms-Impulsive noise, clipping, adaptive filter, NLMS, RLS, QPSK, 16QAM, 32QAM, BER.

\section{INTRODUCTION}

Adaptive Filters with non-stationary statistical characteristics, low cost and their ability to adapt to the unknown environment make them most suitable for the control applications and signal processing [1]. Therefore adaptive filters have been successfully used in numerous signal processing applications over the past decades.

The adaptive filter systems have the same general characteristics i.e. an input signal is received by the adaptive filter and compared with a desired response, generating an error. That error is then used to modify the adjustable coefficients of the filter, generally called filter tap weights, in order to minimize the error . These filters assume that error obtained by adaptive system follow Gaussian distribution.

However practically, noise is impulsive in nature which is non-Gaussian generated by human activities [2], [3] and has more catastrophic effects in communication systems. Nowadays active area of research is to inspect the impulsive noise behavior and suggest solutions to improve the performance of systems by suppressing it. Methods have been proposed to generate impulsive noise such as in [4]. For noise cancellation, clipping technique is implemented in literature which clips the received signal above a threshold [5].

In [6] the performance comparison of the adaptive filter algorithms such as the least mean square (LMS), Normalized LMS (NLMS) and Recursive least squares (RLS) were carried out to remove the noise from an audio signal.

Manuscript received May 4, 2015; revised September 21, 2015.

The authors are with the Department of Electrical Engineering, College of Electrical and Mechanical Engineering (CEME), National University of Science and Technology (NUST), Islamabad, Pakistan (e-mail: sumrin.mehak75@ee.ceme.edu.pk).
Adaptive filters have been used to remove impulsive noise along with comparison with its variants [7]. Impulsive noise has been removed using NLMS filter over different modulation schemes in [8] on the basis of step size and likelihood probabilities.

In this paper, we have presented a hybrid method comprised of the already existing clipping method followed by adaptive filters for impulsive noise cancellation. Clipping method being simple in terms of implementation and carrying out the parameters, yet it can be used for impulsive noise removal along with the adaptive filters, in the presence of AWGN. The proposed method is a hybrid of the clipping method and adaptive filters. In the first step, the data is clipped over a threshold and then in second step, the adaptive filters tracks the original data and the performance of this method is compared with the performance of each of the individual method.

Moreover, a comparison of this proposed method is conducted over different modulation schemes as a contrary to the practical implementation of the noise removal technique. The data is modulated over different types of constellations e.g., QPSK, 16-QAM and 32-QAM.

Following the introduction above, the paper is organized as: Section II briefly describes the basic principle of noise cancellation. Section III gives the review of clipping method which is followed by discussion of different adaptive filters in Section IV, Section V has elaboration of the new proposed method, supported with the simulation results in Section VI. In the end, Section VII concludes the paper followed by the references.

\section{IMPULSIVE NOISE GENERATOR MODEL}

Impulsive noise has been generated using the model given in Fig. 1 in MATLAB/Simulink [2]. It includes the zero-order hold, data source, and sign as a comparator. The output is multiplied by a random number to generate fixed or 1 unit width and variable amplitude impulses. And the frequency response of impulsive noise is a rich signal in terms of frequency as it contains all the frequencies in equal amounts, having a flat response in frequency domain as shown in Fig. 2.

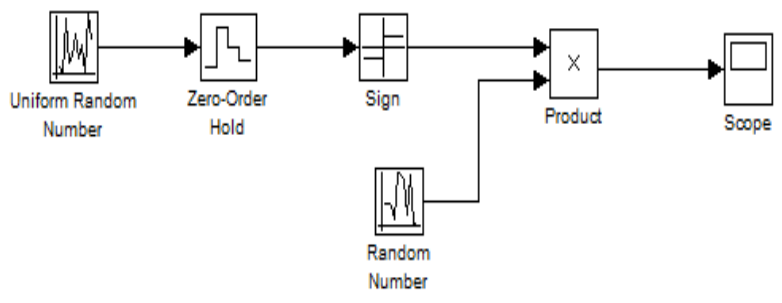

Fig. 1. Impulsive noise generator model. 

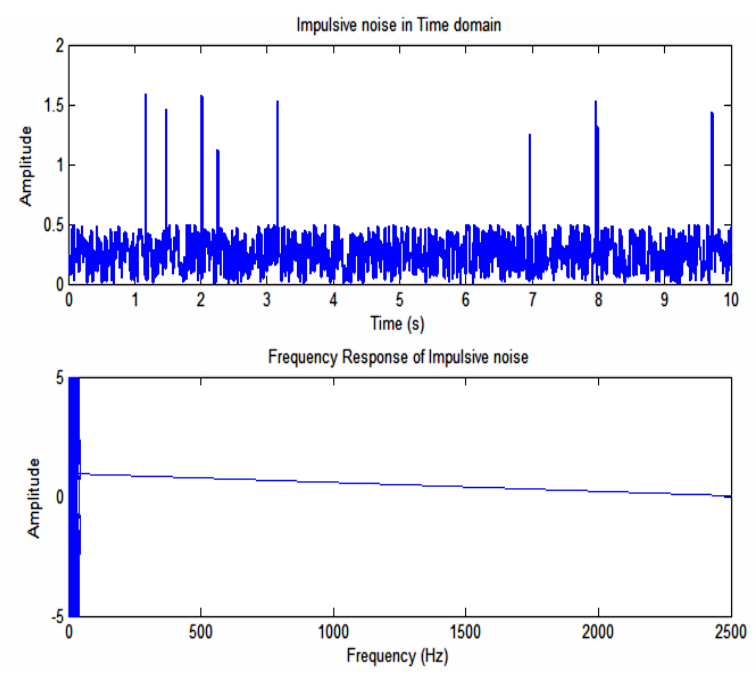

Fig. 2. Impulsive noise generated signal and its spectrum.

\section{ACTIVE NOISE CANCELLATION}

An adaptive filter has an adaptation algorithm, that monitors the environment and vary the filter transfer function accordingly. Each adaptive filter depends on the error signal generated from the adaptive filter to update its filter taps.

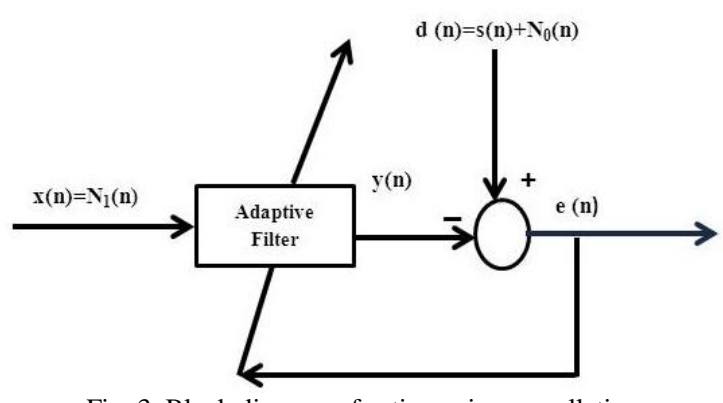

Fig. 3. Block diagram of active noise cancellation.

The output of the adaptive filter is compared with the desired signal $d(n)$. The desired signal is a signal corrupted with another noise source as shown in Fig. 3. The coefficients of adaptive filters adapt recursively to make the error signal $e(n)$ to be minimum so that the output signal $y(n)$ approaches to be equivalent to the desired signal having minimum error. The filter output is obtained as follows:

$$
y(n)=w^{T}(n) x(n)
$$

where $x(n)$ is the filter input and $w(i),(\mathrm{i}=0,1, \ldots, \mathrm{M}-1)$ is the weight vector which corresponds to the filter length $M$. The error signal $e(n)$ involved in the adaptive recursive process, is defined by

$$
\begin{gathered}
e(n)=d(n)-y(n) \\
=s(n)+n_{2}(n)-n_{2}(n)=s(n)
\end{gathered}
$$

\section{CLIPPING}

In practical applications, clipping method is usually used for impulsive noise mitigation due to its simplicity. A clipping algorithm is employed at the receiver end of the AWGN channel, where we presume that impulsive noise is being added by the channel during the communication [9]. It is to reckon that clipping method only changes the amplitude of the data without changing the other parameters such as phase.

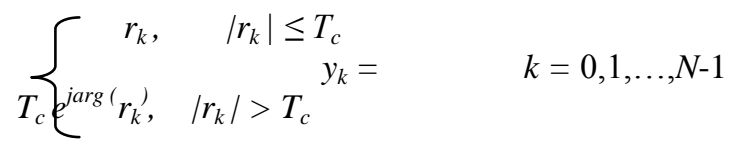

$T_{c}$ is the Clipping threshold that can be set according to the maximum value of data if known. In this method the amplitude of the received data is clipped or limited by the threshold and no other change occurs to the signal/data that has been received.

\section{ADAPTIVE FILTERS}

There are many adaptive algorithms used for noise removal. The brief summaries of adaptive algorithms which are used in this research are as follows.

\section{A. NLMS}

The common variant of Least Mean Square (LMS) algorithm is Normalized Least Mean Square (NLMS) that gives faster convergence than LMS. The basic limitation of the LMS algorithm is its sensitivity to its input signal scaling. The convergence rate is quite slow and step size needs to be chosen carefully to guarantee the stability of the algorithm. The whole algorithm remains the same during the recursive procedure but the filter tap weights are updated using following equation:

$$
w(n+1)=w(n)+\frac{\mu e(n) x(n)}{\epsilon+\left\|x(n)^{2}\right\|}
$$

where $\epsilon$ is a small number added for algorithm stability, $\mu$ is the step size of the filter and $e(n)$ is error signal.

\section{B. $R L S$}

The Recursive least squares (RLS) adaptive filter is the one in which autocorrelation matrix estimation is used to de-correlate the current input data. It recursively finds the filter coefficients that are then used to minimize a weighted linear least squares cost function relating to the deterministic input signals [10]. The filter weights $\mathrm{w}$ are updated in RLS algorithm by following equations.

$$
\begin{gathered}
w(n+1)=w(n)+k(n) x(n) \\
k(n)=\frac{\lambda^{-1} \Phi^{-1}(n-1) x(n)}{1+\lambda^{-1} x^{T}(n) \Phi^{-1}(n-1) x(n)} \\
\Phi^{-1}(n)= \\
\lambda^{-1} \Phi^{-1}(n-1)-\lambda^{-1} k(n) x^{T}(n) \Phi^{-1}(n-1)
\end{gathered}
$$

where $\lambda$ is the forgetting factor. $\Phi^{-1}$ is the cross correlation matrix. The $\lambda$ is initialized with 1 and $\Phi^{-1}$ with $\delta^{-1} I$. Where $I$ is the identity matrix.

\section{PRoposed New Method}

This paper proposes a hybrid technique for impulsive noise 
reduction based on clipping method along with adaptive filters. Fig. 4 shows the simple model carried for the

underlined method.

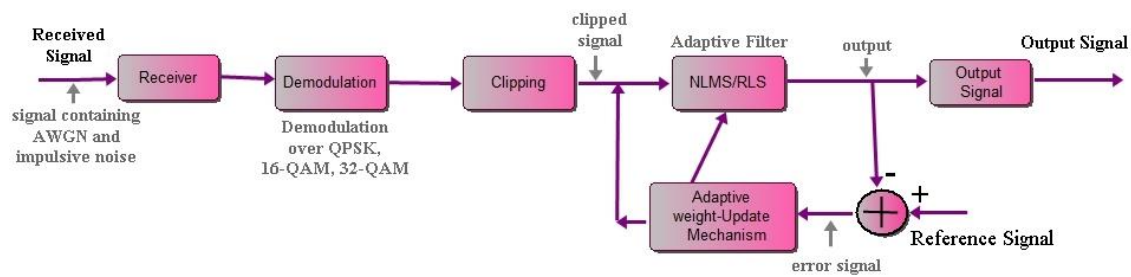

Fig. 4. Block diagram of the proposed method for IN reduction.

The received data is clipped by a defined threshold after demodulation, so as to remove the highest peaks caused due to impulse noise. Here, the clipping method only clips or limits the amplitude of the data and no other parameters or characteristics are changed. Then adaptive filters are used to recover the original data by removing the noise from the data that lie under the threshold used for clipping method, as the clipping method only removes the noise peaks above the threshold.

For an instant, the proposed technique is considered to be a hybrid technique where two different techniques are used in cascade or in combination to each other and is supposed to give better results than each individual method. In this combined method, the higher peaks caused by the impulsive noise are removed by the clipping method then the input to the adaptive filters has lower values and the data can be recovered through the recursive method.

\section{SimULATION RESULTS}

In the first part of simulations, we have tried to recover the binary data perturbed by impulsive noise using clipping method. This is shown by initially generating impulsive noise by following steps mentioned in [2] and depicted in Fig. 2.

The binary data is generated randomly, comprised of 100,000 randomly generated bits, in MATLAB. This binary data is added with the impulsive noise after modulation as shown in Fig. 5 and is then transmitted over an AWGN channel, pretending that impulsive noise has been added through the channel itself during the communication. The unwanted peaks or impulses can be clearly seen above the amplitude of 1 , in the figure below:

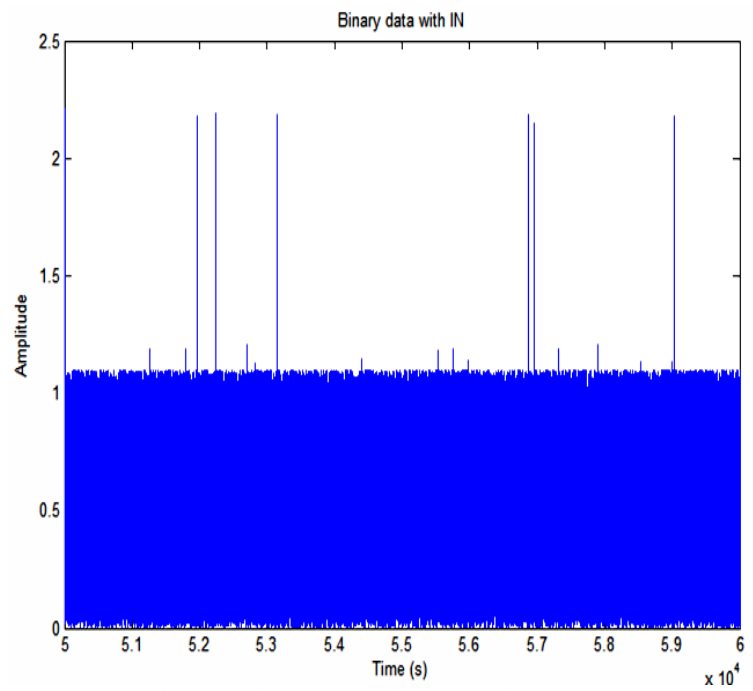

Fig. 5. Original data with impulsive noise.
For clipping method, the received binary data is clipped over a threshold of amplitude 1 after demodulation. In this way the clipping method clips or limits the data above the threshold but it cannot remove the noise that lie below the threshold.

In second part of the simulations, the clipped data is then filtered using the adaptive filters (NLMS \& RLS). The basic scenario is that the output $\mathrm{y}(n)$ of the filter is compared with the desired signal $d(n)$. Their difference produces the error signal $e(n)$ and the filter updates its weights recursively using the adaptive weight update (4) \& (5), such that the error signal is minimized.

In an optimum sense the system output error signal should contain the original signal as in (2). For these simulations the length of the two adaptive filters is fixed to 32. The step size parameter $\mu$ for NLMS Algorithm is chosen to be 0.01 and forgetting factor $\lambda$ for RLS is 0.98 .

Fig. 6 represents the bit error rate (BER) plot of the data after the removal of AWGN and IN using different methods such as the clipping method, NLMS algorithm and NLMS based hybrid proposed method. The performance of these IN reduction methods are compared with the BER plot of the received signal that does not contain impulsive noise and is considered to show the minimum number of errors occurred in the signal, due to the channel itself containing AWGN only.

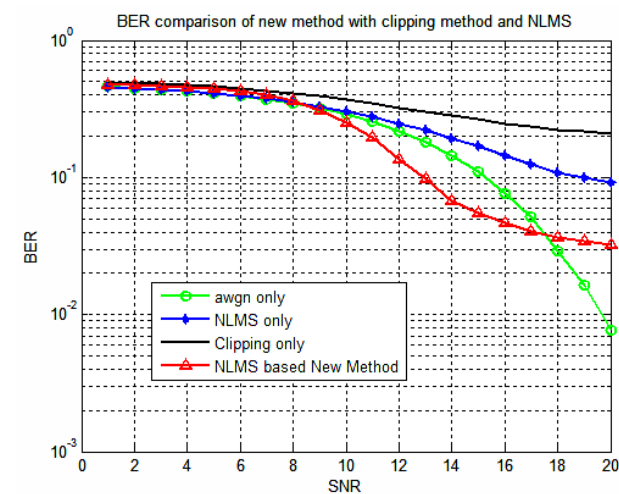

Fig. 6. BER comparison of NLMS based new method with other techniques in AWGN channel.

The BER for the minimum number of errors (without impulsive noise) is the lowest possible BER and it can be seen that among the three algorithms, the performance of proposed method is quite better than the rest of the methods for an SNR of up to $20 \mathrm{~dB}$. It is clear that the performance of NLMS based proposed method degraded by $0.6 \mathrm{~dB}$ while the other two methods performances are degraded by more than 1 dB.

In Fig. 7, the performance of RLS based proposed method 
is compared with clipping and RLS conventional receivers. It is to be noted here that the performance of RLS based proposed method is better than other methods. The performance of RLS based hybrid method is degraded by less than $0.5 \mathrm{~dB}$ while the other methods degrade the performance by more than $1 \mathrm{~dB}$.

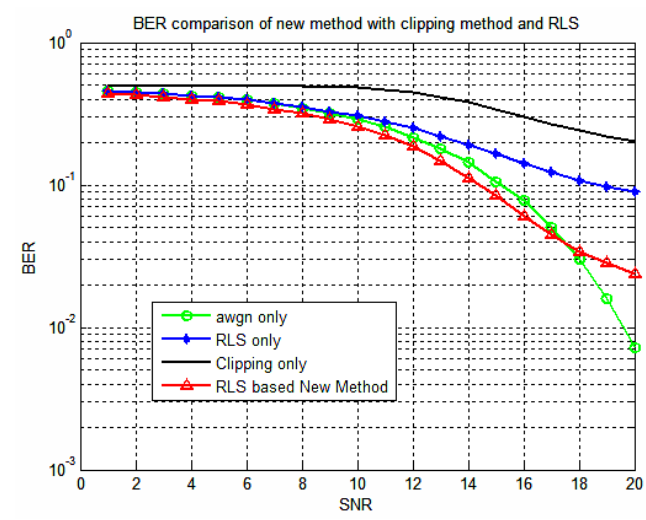

Fig. 7. BER comparison of RLS based new method with other techniques in AWGN channel.

Furthermore, the performance of RLS based proposed method is better than NLMS based proposed method. This is due to the fact that RLS filter belongs to least square family and its recursive method depends on previous samples and cross-correlation matrix. The modulation scheme used for both the results in Fig. 6 \& Fig. 7 is 32 QAM.

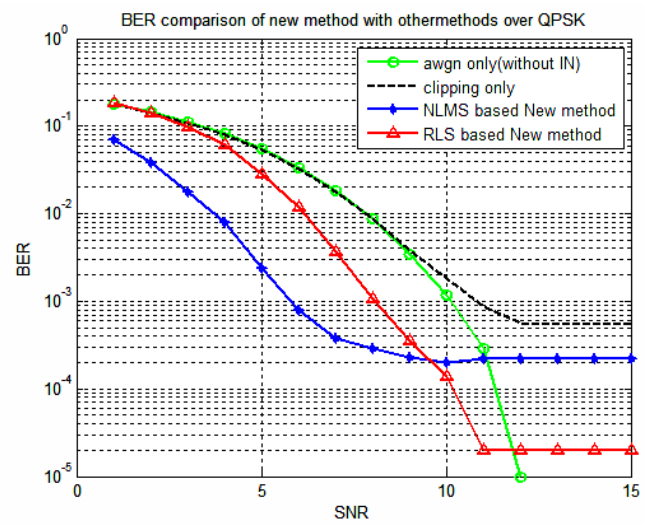

Fig. 8. BER comparison of NLMS and RLS based new method over QPSK in AWGN channel.

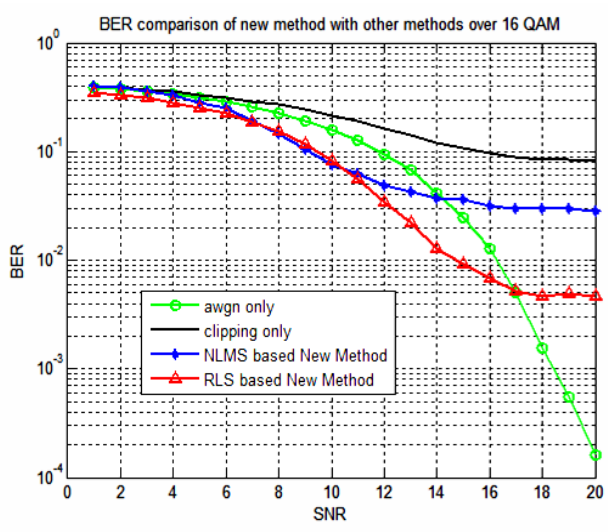

Fig. 9. BER comparison of NLMS and RLS based new method over 16-QAM in AWGN channel.

Also the effect of varying modulation schemes (QPSK, 16-QAM and 32-QAM) on the BER performance of NLMS based proposed method and RLS based proposed method at an SNR level of $20 \mathrm{~dB}$ is investigated and shown in the simulation results of Fig. 8 - Fig. 10. The main aim of this investigation is to find the constellation scheme that gives lower MSE for the new method based on NLMS and RLS adaptive filters.

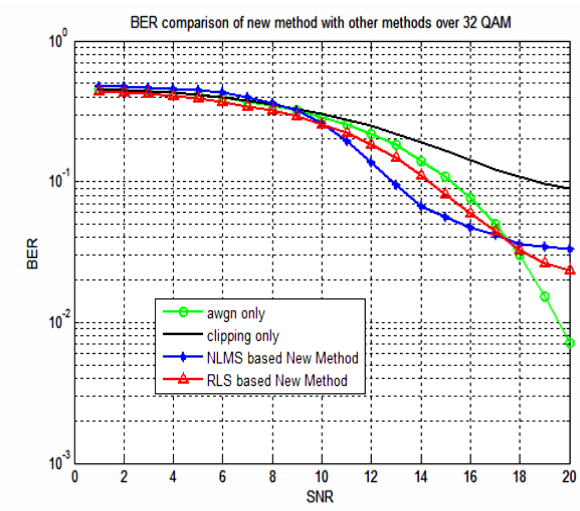

Fig. 10. BER comparison of NLMS and RLS based new method over 32-QAM in AWGN channel.

The performance metric bite error rate in decibel indicates that RLS filter based new method has lower BER compared to other algorithms used. The BER of RLS based hybrid method for the removal of impulsive noise is close to the performance of minimum possible errors having AWGN only and degrades by $0.5 \mathrm{~dB}$ only when compared with other techniques under the same conditions.

\section{CONCLUSION}

In this paper, a Clipping and adaptive algorithms based hybrid method is introduced. The adaptive filters Normalized Least Mean Square (NLMS) and Recursive Least Square (RLS) are used to recover the original data after the received data is clipped over a defined threshold. Due to recursive parameters, the presented scheme exhibits better impulsive noise cancellation when compared with the individual performances of those techniques. Furthermore, the comparison is carried out over different modulation schemes such as QPSK, 16 QAM (square constellation) and 32 QAM (rectangular constellation). It ensured the better performance of the NLMS and RLS based combined or hybrid methods in terms of convergence speed and lower BER, and is verified by the simulation results.

\section{REFERENCES}

[1] B. F. Boroujeny, Adaptive Filters: Theory and Applications, John Wiley, Baffins Lane, Chichester, 1998.

[2] S. R. Al-Araji, M. A. Al-Qutayri, and M. S. Al-Tenaiji, "Impulsive noise reduction using auto-gating technique," in Proc. GCC Conference and Exhibition (GCC), Feb 19-22, 2011, pp. 104-107.

[3] P. Torio, M. G Sanchez, and I. Cuinas, "An algorithm to simulate impulsive noise," in Proc. $19^{\text {th }}$ International Conference on Software, Telecommunications and Computer Networks (SoftCOM), 2011, pp. 1-4.

[4] S. V. Vaseghi, Advanced Digital Signal Processing and Noise Reduction, John Wiley \& Sons Ltd., 4th ed., 2000.

[5] S. Al-Mawali and Z. H Hussain, "Adaptive-threshold clipping for impulsive noise reduction in OFDM-based power line Communications," in Proc. International Conference on Advanced Technologies for Communications, Oct 12-14, 2009, pp. 43-48.

[6] R. K. Thenua et al., "Simulation and performance analysis of adaptive filter in noise cancellation," International Journal of Engineering Science and Technology, vol. 2, no. 9, pp. 4373-4378, 2010. 
[7] B. B. Sawant and S. M. Deokar, "Adaptive filter for removal of impulsive noise and its comparison with LMS algorithm," in Proc. International Conference on Industrial Automation and Computing (ICIAC), April 12-13, 2014, pp. 12-14.

[8] S. A. Jimaa et al., "Impulsive noise reduction using adaptive receiver structure technique," in Proc. IEEE 11th International Conference on Signal Processing (ICSP'12), Beijing, China, vol. 1, Oct 21-25, 2012, pp. 119-122.

[9] S. Haykin, Adaptive Filter Theory, 3rd ed., Prentice Hall, 1996, ch. 9-13.

[10] Recursive least square. [Online]. Available: http://en.wikipedia.org/wiki/Recursive_least_squares

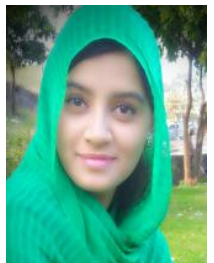

Sumrin Mehak Kabir received her B.E degree from National University of Modern Languages, Islamabad, Pakistan, in 2013. Currently she is pursuing her MS in electrical engineering from National University of Sciences and Technology, Pakistan. Her research interest includes communication systems, signal processing, and information coding. Until now she has 8 publications in different journals and international conferences. She is an electrical engineer (trainee) at Ministry of Water and Power, Govt. of Pakistan.

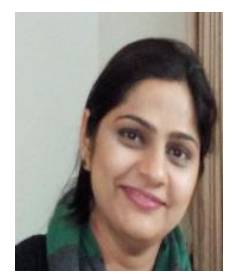

Alina Mirza received her BE degree from University of Engineering and Technology, Taxila, Pakistan, in 2007, the MS electrical degree from National University of Sciences and Technology Pakistan, in 2010. Currently she is pursuing her $\mathrm{PhD}$ from National University of Sciences and Technology, Pakistan in Electrical Engineering. Her research interest includes communication systems, signal processing, information coding and image processing. Until now she has 11 publications in different journals and international conferences. She has been a lecturer in different institutions since 2011 .

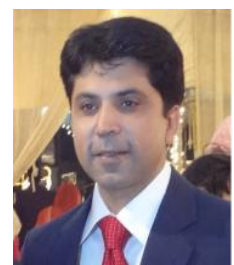

Shahhzad Amin Sheikh received his $\mathrm{PhD}$ degree in electrical engineering from Institute of Mobile Communications, Southwest Jiao Tong University, Chengdu, China in 2006. His research areas are digital signal processing and communication systems. Currently he is the head of the Department of Electrical Engineering in College of Electrical and Mechanical Engineering, National University of Science and Technology, Rawalpindi. 